\title{
Guía de Práctica Clínica: Continúa desaconsejado el uso de terapia de reemplazo hormonal para la prevención primaria de enfermedades crónicas en mujeres posmenopáusicas
}

Moyer VA. Ann Intern Med. 2013;158(1):47-54

\begin{abstract}
Objetivo
Actualizar la recomendación de 2005 de la Fuerza de Tareas Preventiva de los EE.UU. (USPSTF) acerca de la terapia de reemplazo hormonal $(\mathrm{TRH})$ para prevención primaria de enfermedades crónicas en mujeres posmenopáusicas.
\end{abstract}

\section{Fuente y Selección de Datos}

Medline, Cochrane, Scopus y listas de referencias. De los nueve ensayos aleatorizados y controlados publicados que fueron recuperados, el WHI (Women's Health Initiative) fue el único ensayo diseñado para calcular la eficacia de terapia hor- monal (TRH) para prevención primaria de múltiples condiciones crónicas. Este comparó estrógenos equinos conjugados más acetato de medroxiprogesterona y estrógenos solos con placebo en mujeres de 50 a 79 años. Fue el ensayo más amplio, de duración más prolongada y más aplicable a la población objetivo para esta recomendación. Dicho ensayo debió suspenderse por los efectos adversos observados en el grupo de tratamiento con hormonas.

\section{Resultados Principales}

Los principales resultados se resumen en la tabla 1.

Tabla 1. Estimación de la diferencia de tasa de eventos (por 10.000 personas año) asociados al uso de TRH vs placebo en mujeres posmenopáusicas.

\begin{tabular}{|c|c|c|c|c|}
\hline \multirow[b]{2}{*}{ Resultados } & \multicolumn{2}{|c|}{ Estrógenos más progesterona } & \multicolumn{2}{|c|}{ Estrógenos solos } \\
\hline & $\begin{array}{l}\text { Eventos prevenidos } \\
\text { n (IC 95\%) }\end{array}$ & $\begin{array}{c}\text { Eventos causados } \\
\text { n (IC 95\%) }\end{array}$ & $\begin{array}{l}\text { Eventos prevenidos } \\
\text { n (IC 95\%) }\end{array}$ & $\begin{array}{c}\text { Eventos causados } \\
\text { n (IC 95\%) }\end{array}$ \\
\hline \multicolumn{5}{|l|}{ Beneficios } \\
\hline Fracturas totales & 46 (29 a 63) & - & 56 (37 a 75) & - \\
\hline Fracturas de cadera & $6(1$ a 10$)$ & - & 7 (1 a 12) & - \\
\hline Incidencia de ca de mama invasor & - & - & $8(1$ a 14$)$ & - \\
\hline Muertes por ca de mama & - & - & 2 (1 a 3) & - \\
\hline \multicolumn{5}{|l|}{ Daños } \\
\hline Accidente cerebrovascular & - & $9(2 \mathrm{a} 15)$ & - & 11 (2 a 20) \\
\hline Trombosis venosa profunda & - & 12 (6 a 17$)$ & - & 7 (1 a 14) \\
\hline Trombo embolismo pulmonar & - & $9(4$ a 14$)$ & - & - \\
\hline Ca. Mama invasor & - & $8(3$ a 14$)$ & - & - \\
\hline Colelitiasis & - & 20 (11 a 29) & - & 33 (20 a 45) \\
\hline Demencia & - & 22 (5 a 39) & - & - \\
\hline Incontinencia urinaria & - & 872 (591 a 1153) & - & 1271 (883 a 1660) \\
\hline
\end{tabular}

\section{Conclusiones}

La TRH utilizada para la prevención primaria de enfermedades crónicas en mujeres posmenopáusicas presenta riesgos que superan ampliamente los beneficios. La USPSTF recomienda no utilizarla para este fin (recomendación tipo D).

\section{Comentario}

La TRH es probablemente uno de los temas de salud que más artículos, editoriales y notas de prensa han suscitado en los últimos años. Los primeros trabajos epidemiológicos, en la década de los 80 ' y los 90', sugerían que el uso de estrógenos artificiales podía, además de aliviar los síntomas de la menopausia, proteger de la enfermedad coronaria y otros trastornos graves. Sin embargo, el ensayo clínico HERS constató que la TRH combinada no es mejor que el placebo en la prevención de patología coronaria'. Posteriormente, el estudio WHI mostró que el uso de estas hormonas, en combinación con progestágenos, aumenta la incidencia de cáncer de mama, así como de tromboembolismo venoso, accidentes cardiovasculares y cerebrales ${ }^{2}$. Si bien está demostrado que los daños asociados a la TRH superan a los beneficios, este tratamiento no debe quedar etiquetado como un "veneno", ya que puede resultar de utilidad en algunas instancias (por ejemplo, alivio sintomático de sofocos refractarios a otros abordajes, menopausia precoz). Aún todavía en este grupo seleccionado de mujeres donde podría tener indicación la terapia hormonal no está bien definido por cuanto tiempo, qué dosis o qué tipo de presentación hormonal sería la mejor indicación. Algunos tipos de TRH tienen mayor riesgo; y los riesgos propios de cada mujer pueden variar dependiendo de su historia de salud y estilo de vida. La FDA de los Estados Unidos está aconsejando por ahora a las mujeres menopáusicas que usen hormonas que lo hagan por el tiempo más corto posible y en la dosis más baja posible para controlar los síntomas.

\section{Conclusiones del comentador}

Hay otras opciones, además de la TRH, que alivian los síntomas menopáusicos y que ayudan a prevenir y tratar la osteoporosis. Es importante "normalizar" la menopausia como una etapa más de la vida de la mujer.

Nadia Silvina Musarella [ Servicio de Medicina Familiar y Comunitaria del Hospital Italiano de Buenos Aires. nadia.musarella@hiba.org.ar]

Musarella N. Continúa desaconsejado el uso de terapia de reemplazo hormonal para la prevención primaria de enfermedades crónicas en mujeres posmenopáusicas. Evid Act Pract Ambul Oct-Dic 2013; 16(4):152. Comentado de: Moyer VA. Menopausal Hormone Therapy for the Primary Prevention of Chronic Conditions: U.S. Preventive Services Task Force Recommendation Statement. Ann Intern Med. 2013;158(1):47-54. PMID: 23090711.

\section{Referencias}

1) Group for the Women's Health Initiative Investigators. Risk and benefits of estrogen plus progestin in healthy postmenopausal women. JAMA 2002; 288:321-3

2) Grady D, Herrington D, Bittner V, et al. Cardiovascular disease outcomes during 6,8 years of hormone therapy. Heart and estrogen/progestin replacement study follow-up (HERS II).JAMA 2002; 288:49-57. 